\section{El fundamento científico de la \\ función de filtro del médico \\ general}

\section{The scientific basis for the gatekeeping role of general practicioners}

\author{
Juan Gérvas* \\ Mercedes Pérez Fernández \\ Equipo CESCA - Madrid, España
}

Declaración de conflicto de intereses: Los autores declaran no tener ningún conflicto de interés en lo que respecta a este texto.

Declaración respecto a aspectos éticos: Este es un trabajo de investigación sobre datos secundarios de acceso público, que no implica experimentación en ninguna forma. No hay, pues, ningún problema ético al respecto.

*E-mail: jgervasc@meditex.es

\section{Resumen}

Los servicios sanitarios cuentan con niveles de atención, lo que optimiza los resultados. Los niveles tienen "filtros" que aumentan la prevalencia de enfermedad entre los pacientes que llegan a niveles sucesivamente altos. En este trabajo se justifica la existencia del filtro del médico general con respecto al especialista y al hospital por el aumento de la prevalencia de enfermedad en la población derivada de primaria a hospitalaria. Se utilizan ejemplos empíricos respecto al dolor abdominal, dolor precordial y hemorragia rectal. En este último ejemplo, la prevalencia del cáncer de recto y de sigma pasa del $0,1 \%$ en la población al $2 \%$ en la consulta del médico general (por efecto del filtro personal y familiar) y al 36\% en la consulta del especialista (por efecto del filtro del médico general). La selección aumenta el valor predictivo positivo de las pruebas diagnósticas que solicita el especialista, y evita el contacto innecesario con los especialistas a muchos pacientes con hemorragia rectal por causa benigna, que permanecen en su nivel (de la familia, o del médico general).

Palabras clave: Filtro. Atención Primaria. Médico general 
Health services are organized by levels of care, which improves outcomes. These levels are "filters" that lead to a progressive increase in the prevalence of diseases in higher levels of care. We studied and justified the role of the general practitioner as filter, or gatekeeper, to specialists and hospitals. This role leads to greater prevalence of diseases in the referral population. We analyzed empirical data on abdominal pain, chest pain and rectal bleeding as examples. As to rectal bleeding, the prevalence of rectum and sigma carcinomas increases from $0.1 \%$ in the population, to $2 \%$ in general practitioners' waiting rooms (as a consequence of personal and family filters), and to $36 \%$ in specialists' waiting rooms (as a consequence of the gate-keeping role general practitioners play). This increase in prevalence improves the positive predictive value of the specialist's diagnostic testing, and patient selection avoids unnecessary contacts of specialists with patients having benign causes of rectal bleeding (who remain at their appropriate family or general practitioners' level).

Key words: Gatekeeping. Primary Care. General Practitioner/Family physician

\section{Introducción}

Los problemas de salud ${ }^{\text {a }}$ exigen respuestas, pues se viven con preocupación. Muchas veces conllevan sufrimiento, y no es extraña la sensación de amenaza vital, de fragilidad personal. Todo ello exige tomar decisiones para intentar cambiar el curso de los acontecimientos y restaurar la salud. Tomar decisiones no es, siempre, buscar al médico y seguir el proceso diagnóstico y terapéutico que éste proponga. Hasta llegar al médico suele haber un largo camino.

El paciente decide sobre el nivel a que se deba requerir atención con las percepciones simultáneas acerca de (1) la "normalidad", (2) la posible ayuda que pueda prestar el sistema sanitario y (3) la experiencia personal y en el entorno familiar, laboral y social. Así, mientras un vómito tras una comida excesiva se suele resolver en casa y sin intervención sanitaria alguna, un vómito de sangre suele llevar al paciente a urgencias hospitalarias. El vómito de alimentos se interpreta como una respuesta normal del organismo, y se resuelve con cuidados familiares, o personales, a domicilio (una infusión de poleo, un cierto grado de ayuno, comidas suaves, y poco más). El vómito de sangre, sobre todo si es abundante, espanta al paciente y a su familia, que busca socorro en urgencias, habitualmente. Por supuesto, se puede renunciar a la atención médica urgente, si el paciente tiene experiencia previa de estos cuadros de hematemesis (por ejemplo, por cirrosis hepática grave) y discrepa de los métodos diagnósticos y del éxito de la terapéutica.

En cualquier caso, los pacientes suelen saber cómo moverse en la jungla de servicios sanitarios, al menos para discriminar si requieren cuidados profesionales $\mathrm{y}$, por ejemplo, si se quedan con el médico general o si pasan a mayores y van a urgencias hospitalarias.

${ }^{a}$ Es problema de salud todo aquello que requiere, o puede requerir, una acción por parte del médico o del agente de salud (paciente incluido). En una definición más académica, es problema cualquier queja, observación o hecho que el paciente o el médico perciben como una desviación de la normalidad, que ha afectado, afecta o puede afectar la capacidad funcional del paciente. Son ejemplos de problema de salud: una enfermedad, un síndrome, un signo, un síntoma, un factor de riesgo, una alteración familiar, laboral o social, una deficiencia, una incapacidad, una minusvalía, etc. Problema de salud es algo que requiere una acción sanitaria, algo que preocupa por no verse como normal. 


\section{De los cuidados informales a los cuidados profesionales}

La toma de contacto con el médico general cambia la dimensión del problema, pues pasa de una fase de auto-cuidado a otra de cuidados profesionales. Lo importante sigue siendo la persona en su entorno, y se maneja todavía mucha información blanda, pero el modelo científico biológico tiende a imponer "el caso" y los datos "duros"b

El médico general puede enfocar el problema como una alteración esperable de la normalidad, o como una variación de la misma, lo que tranquilidad y sosiega si se explica bien al paciente y sus familiares. También puede adoptar una "espera expectante", tras la valoración del problema, y de acuerdo con el paciente. En otro caso, el médico general puede decidir iniciar un tratamiento que lleve al alivio de los síntomas, si se ha formado una opinión suficiente a través de la entrevista clínica y la exploración física, y con el conocimiento previo del paciente y de su entorno.

Si el médico general decide iniciar un proceso diagnóstico puede solicitar directamente las pruebas, y después (o de entrada) derivar al paciente a un especialista, si juzga que son necesarios los conocimientos del mismo (por ejemplo, para el diagnóstico y tratamiento de una probable estenosis de la arteria renal). También puede derivar al especialista si cree que son convenientes para el paciente sus habilidades terapéuticas (por ejemplo, el paciente con un problema de auto-imagen, por unas orejas “en asa”). Si a la necesidad de derivación se une la impresión de gravedad y de urgencia, el paciente es derivado al servicio de urgencias hospitalarias, a veces con sólo un diagnóstico probable, que justifica la derivación ("paciente con suave disnea de comienzo brusco, con taquicardia de 120 , algún estertor en base derecha, desdoblamiento del segundo tono, no dolor ni fiebre, intervenido hace tres semanas de prostatectomía; sospecha de embolia pulmonar").
La derivación al especialista da otra dimensión al problema, y suele alejar al paciente de su entorno geográfico cercano, especialmente si incluye la hospitalización, o derivación a un hospital terciario (docente). Además, el modelo científico biológico se impone con toda su fuerza y el paciente tiende a convertirse en "un caso", y se suele utilizar información dura casi en exclusiva ${ }^{1}$. Con el especialista lo que prima es la resolución de la incertidumbre y la búsqueda de todo posible diagnóstico. El tiempo diagnóstico se alarga, excepto cuando la derivación es a los servicios de urgencia. Todo ello se justifica si es necesario, cuando llegar al especialista tiene más beneficios que perjuicios esperables, y si el diagnóstico y la terapéutica mejoran el pronóstico del paciente ${ }^{2}$. Pero se convierte en un simple absurdo si no se justifica, si fue innecesario.

\section{Sobre la importancia del filtro personal y familiar}

La existencia del agente de salud familiar sereno y sensato es clave para el paciente (y la familia), y para el funcionamiento adecuado de los sistemas sanitarios. Por ejemplo, el esquizofrénico que cuenta con una madre sensata y lógica, capaz de utilizar adecuadamente los recursos sanitarios y de reconocer y resolver las situaciones crónicas y agudas que cambian de continuo, puede vivir su enfermedad en forma totalmente diferente a otro paciente esquizofrénico sin una madre tal, con un cuadro clínico similar, y una familia semejante en lo que respecta a composición, educación y recursos económicos. En otro ejemplo, la madre que cría tres niñas y resulta incapaz de resolver por sí misma los síntomas menores que surgen con catarros, traumatismos y otros problemas de salud, consume más de diez veces los recursos sanitarios que utiliza otro familia similar en todo, excepto en la sensatez, serenidad y buen hacer de la madre. Un último ejemplo, lo que supone un cuidador principal entero y lógico

bSon datos duros los que se obtienen a través de "máquinas", de pruebas instrumentales. Por ejemplo, el peso, la talla, el electrocardiograma, la radiografía de tórax, la cifra de glucemia, el recuento de hematíes, etc. 
(generalmente mujer) para el paciente terminal, que puede llegar a morir en paz en su domicilio a poco que encuentre un médico general colaborador; en otro caso morirá en una habitación desangelada de hospital, en un pasillo de urgencias, o incluso en una ambulancia, camino del hospital.

La importancia del filtro personal y familiar se demuestra en el caso de la hemorragia rectal. Este signo es muy frecuente en la población, ya que tiene un incidencia anual del $20 \%^{3,4}$. Pero quienes presentan este signo no acuden inmediatamente y en masa al médico, pues antes de consultar con el médico general, en torno al $80 \%$ lo comenta con algún familiar o amigo, y un 30\% compra y ensaya el efecto de un preparado anti-hemorroidal $^{4}$. De hecho, los que solicitan cuidados profesionales se "filtran" muy bien, pues consultan más frecuentemente con su médico general los mayores de 60 años y los que ven la sangre mezclada con las heces (comparados, respectivamente, con los de menor edad, y con los que advierten la sangre sobre las heces, o simplemente en el papel higiénico). Aproximadamente, consulta con el médico un $41 \%$ de los que tienen rectorragia alguna vez en la vida, y lo hacen a unos dos meses del inicio del signo, de media ${ }^{4}$.

Con este conjunto de sabias medidas, la prevalencia de cáncer de recto y sigma asociada a la rectorragia pasa de ser del uno por mil en la población al veinte por mil entre los que consultan con su médico de atención primaria $^{3}$. Es decir, la eficacia del filtro personal y familiar multiplica por veinte la prevalencia de rectorragia asociada a cáncer de recto y sigma entre los pacientes que llegan a la atención primaria. Con ello se facilita el trabajo del médico general, se aumenta el valor predictivo de las actividades diagnósticas, y se utilizan adecuadamente los recursos sanitarios.

Así pues, el filtro personal y familiar evita el contacto innecesario de miles de pa- cientes con el sector sanitario profesional. No es sólo que disminuya la carga de trabajo y haga posible el uso racional de los recursos, es, sobre todo, que no se "medicalizan" situaciones normales y "anormalidades normales”. Situaciones que nunca disminuirán la salud ni alterarán la calidad de vida del paciente, excepto si pasan de los cuidados personales y familiares a los cuidados profesionales, por el riesgo de cristalización que ello conlleva (más los asociados a las múltiples cascadas diagnósticas y terapéuticas que conllevan el cuidado profesional $)^{5}$. De hecho, se calcula que sólo llegan a los profesionales la cuarta parte de los problemas de salud que se presentan en la población ${ }^{6,7}$.

\section{La beneficiosa concatenación del trabajo del médico general y del especialista}

El médico general distingue mejor que el especialista a los sanos de los enfermos y por ello es fundamental el trabajo en cadena, con el médico general de filtro de la demanda que ha de pasar a consultar al especialista ${ }^{8}$. Médicos generales y médicos especialistas son complementarios.

Si suponemos que los enfermos son manzanas de una cesta recién llegada al mercado, el médico general es capaz de sacar de la cesta las sanas, con eficacia y rapidez, y casi sin confundirse. No obstante, habrá alguna manzana podrida entre las sanas seleccionadas, algún "falso negativo" [el médico general tiende a ver como sanas a algunas manzanas podridas, pero si ofrece longitudinalidad ${ }^{c}$ corrige el error al verlas repetidamente, en días sucesivos].

Al pasar la cesta al especialista, irán sanas y podridas, pero la densidad de sanas será mucho menor que al comienzo del proceso. Así se disminuye la posibilidad de errar del especialista, que tiende a ver la

'Es longitudinalidad la prestación de cuidados a lo largo del tiempo y para los distintos problemas de salud. Es continuidad la prestación de cuidados para un problema de salud. Por ejemplo, es continuidad la concatenación de eventos de atención en un paciente diabético, y es longitudinalidad la concatenación de eventos de atención para todos los problemas de salud de este paciente diabético a lo largo del tiempo. Un especialista puede ofrecer continuidad de la atención pero no longitudinalidad de la misma. Un médico general ofrece continuidad y longitudinalidad. 
manzanas sanas como podridas. Si el especialista accede a la cesta sin el trabajo de selección del médico general, con una mayoría de manzanas sanas, tenderá a cometer muchos errores, por clasificar a muchas como podridas (falsos positivos), y en el proceso para aclarar la enfermedad que les afecta acabarán pudriéndose ${ }^{5}$. Además, el especialista sólo sabe de un determinado tipo de enfermedades (las de su especialidad), de manera que el médico general debe eliminar las manzanas sanas de la cesta, y preparar una cesta para cada especialista, donde vayan las manzanas probablemente podridas de su especialidad. De nuevo, si el especialista recibe directamente la cesta, además de considerar a muchas manzanas como podridas, tenderá a verlas afectadas por enfermedades de su especialidad, a diagnosticarlas y tratarlas dentro de su propio campo de trabajo.

En un símil ya empleado ${ }^{9}$, el médico general selecciona a los pacientes en el Mar de la Incertidumbre, y los desembarca en distintos puertos de la Tierra de los Síntomas, cuando lo estima necesario. Los especialistas de cada puerto, cíclopes con una lente por ojo único, diagnostican y tratan pacientes con (1) un bajo umbral diagnóstico (casi siempre emprenden actividades diagnósticas), (2) un largo recorrido diagnóstico (invierten en ello mucho tiempo, con diversas pruebas y alto coste), y (3) un alto umbral terapéutico (precisan de mucha información para decidirse a tratar). Su travesía por el Mar de la Incertidumbre es larga, y lleva al paciente a lugares pre-determinados (acordes con la especialidad) del Reino de los Diagnósticos y del Imperio de los Tratamientos. Por ello el médico general debe ser cuidadoso en la derivación de los pacientes, que idealmente sólo estaría justificada cuando se derivara al especialista idóneo para el tipo de problema del paciente y cuando las actividades del especialista proporcionaran más beneficios que perjuicios.

En la consulta de Medicina General, el médico debería resolver la amplísima mayoría de los problemas de salud de los pacientes por sí mismo, ya que ofrece un re- corrido diagnóstico más corto y con mayor variedad de alternativas diagnósticas y terapéuticas que el especialista. En muchos casos, no hay más que valorar y tranquilizar respecto a que se trata de un problema de variación de la normalidad, o de "anormalidad normal". Si no puede resolver el problema, el médico general debería derivar con acierto, respecto al momento y destino adecuado.

En Europa, el médico general tiene el monopolio de las "primeras consultas" y actúa de filtro para la atención especializada en Dinamarca, España, Irlanda, Italia, Holanda, Portugal y el Reino Unido. Desde 2004 se ha implantado en Alemania y Francia un mayor pago al médico especialista que recibe al paciente a través del médico general, lo que de facto está dando también el papel de filtro a los médicos generales alemanes y franceses.

\section{El dolor abdominal como caso acerca de los beneficios de la función de filtro del médico general}

En Medicina hay mucho problemas de salud autolimitados. Es, por ejemplo, el dolor por la hernia discal lumbar, que tiene un curso relativamente benigno en el que muchas de ellas, sintomáticas o silentes, se reabsorben espontáneamente (hasta el $50 \%)^{10}$. En estos problemas autolimitados se puede estudiar la función de filtro del médico general, pues es clave mantener alejados a los pacientes de los especialistas para que la actuación médica esté justificada respecto al balance de beneficios y riesgos, y para que no haga "cristalizar" el proceso y provoque el desarrollo y fijación de la conducta de enfermo ${ }^{5}$.

El dolor abdominal es uno de los típicos problemas de salud autolimitados, que ser resuelven espontáneamente con el curso del tiempo. El dolor abdominal es un buen ejemplo para estudiar la beneficiosa concatenación del trabajo del médico general y del especialista. Es un síntoma frecuente, con una prevalencia en España del 3,2 \% 
como razón de consulta ${ }^{\mathrm{d}}$ nueva para el encuentro con el médico general, sólo por detrás de la tos, la fiebre y las molestias de garganta $^{11}$. Su pronóstico es relativamente benigno, ya que se resuelve por sí mismo con el simple paso del tiempo en más de la mitad de los casos, aunque puede asociarse a eventos graves, como apendicitis en el $0,7 \%$ , cáncer de aparato digestivo en el 0,8\%, cáncer de aparato genital femenino en el $0,3 \%$, o cáncer del aparato respiratorio en el $0,2 \%$ de los casos ${ }^{11-14}$.

El médico general debería ser capaz de seleccionar entre los pacientes que acuden por dolor abdominal a (1) los que no tienen causa definida, y sólo necesitan una valoración, sin ninguna solicitud de prueba diagnóstica ni ninguna prescripción de medicamentos, (2) los que precisan ser sometidos a actividades diagnósticas y/o tratamientos farmacológicos en el entorno de la Medicina General, (3) los que deben ser derivados al especialista, por un problema agudo o crónico que requiere actividades diagnósticas y terapéuticas propias del segundo nivel y (4) los que habiendo sido ya estudiados por los especialistas deben seguir siendo atendidos, bien en ese nivel, bien por el propio médico general.

Aunque es una cuestión frecuente en Medicina General, desconocemos con precisión el proceso de atención al paciente con dolor abdominal, excepto en algún caso concreto bien estudiado, que aun no siendo generalizable resulta útil para su análisis. Los médicos generales deberíamos ser especialistas de lo frecuente, y producir conocimiento al respecto, pero también en esto nos deslumbran los especialistas, y la cuestiones importantes siguen siendo ignoradas, por mucho que constituyan el trabajo nuestro de cada día. Tenemos escasa investigación acerca de lo frecuente, y menos aún relativa a la longitudinalidad (la atención a lo frecuente en pacientes reales, con problemas múltiples, y con encuentros varios y enlazados respecto a dichos problemas).

Sobre al dolor abdominal, sirva de ejemplo el seguimiento de 150 pacientes consecutivos atendidos con dolor abdominal de nueva presentación por tres médicos generales ingleses durante ocho meses de trabajo con una población urbana de 8.600 personas ${ }^{13}$. La prevalencia anual de consulta por este motivo fue, pues, del 2,6\%.

Los pacientes acudieron por dolor abdominal de muy distinta duración, desde menos de 24 horas (el 16,7\%) a más de seis meses (el 6\%), siendo dos días la duración media del dolor antes de acudir al médico general.

En el análisis del proceso de la atención, al cabo de un año, se demostró que los médicos generales atendieron al $89 \%$ exclusivamente en el entorno de la Medicina General. Derivaron al 11\%. De los 17 pacientes derivados, 10 lo fueron a urgencias.

$\mathrm{Al}$ año se pudo valorar que habían requerido realmente la atención especializada el $59 \%$ de los derivados. No hubo ningún falso negativo (ningún paciente fue erróneamente etiquetado como "no precisa atención especializada").

A la mitad de los pacientes con dolor abdominal se le atendió con tan sólo valoración (historia clínica y exploración física), consejos varios y tranquilización acerca del carácter autolimitado del problema. Al 43\%, con alguna medicación. Y al 17\% con algún estudio diagnóstico, como recuento leucocitario en sangre, tira de orina, radiología con contraste de bario y demás.

En el 14\% no se llegó a ningún diagnóstico al cabo del año, ni siquiera los muy inespecíficos diagnósticos que recibieron algunos, como "colon irritable", "infección vírica”, o "dispepsia”. Estos 21 pacientes curaron sin problemas.

Hubo 12 casos en los que se cometió error diagnóstico, desde el simple atribuir el

dEs razón de consulta la causa que desde el punto de vista del paciente justifica el encuentro con el médico. En una definición más académica, es el enunciado, aceptado por el paciente, de la razón de su contacto con el sistema sanitario; dicha razón representa la demanda de atención del paciente. La razón de consulta puede ser un síntoma (dolor abdominal), un signo (erupción cutánea), una enfermedad (revisión de su diabetes), un temor (miedo al cáncer de pulmón), una cuestión administrativa (un certificado de salud), un problema social (pobreza), y, en general, lo que lleva al paciente a tomar contacto con su médico. 
dolor abdominal a una infección urinaria que no se demostró, hasta una sospecha en falso de apendicitis (fue una gastroenteritis), y una apendicectomía innecesaria [se realizaron dos, una con apéndice normal en el estudio anatomopatológico]. Entre los diez pacientes derivados a urgencias se ingresaron a nueve, de los que cuatro tuvieron una hospitalización innecesaria.

Entre los 133 pacientes seguidos exclusivamente en el entorno de la Medicina General, al cabo del año, el 94\% se había recuperado completamente de su dolor abdominal, y sólo en un paciente tenía más molestias que al comienzo (pero sin aparentemente requerir atención especializada).

Si suponemos que las manzanas son pacientes con dolor abdominal, en este caso la cesta de los médicos generales contenía 150 manzanas. Tras valorar a todas, se derivaron al especialista 17.

La cesta que se remitió a los especialistas contenía 7 manzanas sanas, o que no hubieran requerido el contacto con el especialista (falsos positivos). Es decir, dicha cesta contenía los 10 únicos pacientes del total de la población que a lo largo del año se demostró precisaban la atención especializada (verdaderos positivos). Visto desde otro punto de vista, al cabo del año, los médicos generales no cometieron ningún error por omisión (no se quedaron con ningún falso negativo, pacientes que hubieran requerido el cuidado de especialistas).

Los médicos generales supieron seleccionar entre los 150 pacientes a los 10 que precisaban atención especializada, y para ello remitieron una cesta seleccionada de 17 manzanas a los especialistas, con una densidad del 59\% de verdaderos positivos. Dicho de otra forma, los médicos generales evitaron la visita al especialista de una amplia mayoría de pacientes sanos (el 95\%, los verdaderos negativos).

En el proceso de atención a las diez urgencias, los especialistas clasificaron erróneamente a cuatro pacientes ( $40 \%$ ), que fueron innecesariamente ingresados.

Los médicos generales transformaron una necesidad de cuidados especializados del 7\%, en la población que les consultó en una necesidad de cuidados especializados del $59 \%$ en la cesta de manzanas remitida a los especialistas. Es decir, multiplicaron por más de ocho la prevalencia de necesidad de cuidados especializados en la muestra que derivaron. Aprovecharon la longitudinalidad para corregir el error inicial aparente de no remitir tres casos a urgencias en el primer encuentro, pero de estos tres pacientes sólo uno requirió realmente la atención especializada.

Si aceptamos que los especialistas cometen un error del $40 \%$ en la atribución de necesidad de cuidados especializados cuando atienden pacientes en urgencias, derivados por su médico general (cuatro errores respecto a las diez manzanas que se les remitieron en la cesta), podemos calcular que el acceso directo de todos los pacientes con dolor abdominal a las urgencias hospitalarias hubiera conllevado la aplicación de cuidados innecesarios especializados a unos 56 pacientes de los 140 que no necesitaban los cuidados especializados. Esta cifra es conservadora, pues los médicos generales no derivaron "en general" y sólo a urgencias, sino a especialistas acordes con la probable necesidad del paciente, desde ginecología a pediatría, cirugía y psiquiatría, lo que no resultaría fácil para los propios pacientes. Es necesario, pues, que el médico general trabaje como filtro, si se quiere evitar el encarnizamiento de los especialistas con los pacientes sanos.

\section{El dolor abdominal en la consulta del médico general y en la del especialista}

La extrapolación desde la atención primaria a la especializada es, ciertamente, un ejercicio riesgoso. Lo contrario, también (aunque se hace muy frecuentemente). Las poblaciones que atienden generalistas y especialistas son muy diferentes, y el proceso de derivación introduce cambios que requieren ser considerados específicamente en cada caso. Por ejemplo, en Medicina General mejoran o curan el $68 \%$ de los pacientes 
con dolor abdominal crónico sin causa orgánica al cabo de un año, mientras ese porcentaje baja hasta el $12 \%$ en pacientes con el mismo cuadro clínico aparente atendidos en consultas externas hospitalarias ${ }^{12}$.

En la consulta del médico general la probabilidad de que un dolor abdominal sea apendicitis aguda es baja, algo menos del $1 \%{ }^{13}$. En el estudio inglés citado, se atendieron 150 pacientes con dolor abdominal agudo y crónico, hubo sospecha de apendicitis en dos casos, y los dos casos se derivaron a urgencias (la prevalencia de sospecha de apendicitis aguda en la muestra de 10 pacientes derivados a urgencia por dolor abdominal agudo fue del 20\%). En urgencias se descartó una apendicitis (resultó una gastroenteritis). Se realizaron dos apendicectomías, y sólo en un caso se confirmó la apendicitis aguda con la anatomía patológica.

En un estudio diferente, suizo, también en la década de los ochenta del siglo pasado, se analizó el proceso de atención a los pacientes con dolor abdominal sin relación con traumatismos en el servicio de urgencias de un hospital quirúrgico universitario ${ }^{15}$. En los ocho meses de registro se atendieron 241 pacientes con dolor abdominal. Fueron remitidos por sus médicos con sospecha de apendicitis aproximadamente el $20 \%$. Se confirmó el diagnóstico de apendicitis en la mitad de este grupo "sospechoso".

Respecto al total de pacientes, hubo 48 casos de apendicitis agudas confirmadas; es decir, el $20 \%$ de los diagnósticos finales en pacientes atendidos en urgencias hospitalarias quirúrgicas con dolor abdominal sin relación con traumatismos, pero sólo la mitad procedieron de la muestra de pacientes con sospecha de apendicitis como causa de derivación a urgencias.

Se realizaron 54 apendicectomías. Entre los pacientes operados la anatomía patológica confirmó el diagnóstico en el 89\%. La tasa de un once por ciento de errores en las apendicectomías es muybuena, pues se suele aceptar una tasa de extirpación de apéndices normales por sospecha de apendicitis aguda en torno al veinte por ciento [la incorporación de algoritmos como el de Alvarado, o de la ecografía con compresión, no mejora demasiado las cosas ${ }^{16}$.

Adicionalmente, aproximadamente se dio de alta al $10 \%$ de los pacientes sin llegar a un diagnóstico, y con buen estado de salud ${ }^{15}$.

Respecto a las apendicitis, pues, el médico general suele recibir una caja de manzanas con una prevalencia en torno al $1 \%$ entre pacientes con dolor abdominal, y en la caja de manzanas que deriva a urgencias del hospital por dicho dolor suele seleccionar todos los casos de apendicitis, con una prevalencia en torno al $20 \%$. Al final, las manzanas con apendicitis confirmada por la anatomía patológica son también el 20\% de las que llegan a urgencias hospitalarias por dolor abdominal, pero sólo la mitad proceden de la muestra en la que el médico general sospechó la apendicitis. La otra mitad estaba en la caja de manzanas derivadas a urgencias, pero con otras etiquetas, o sin ninguna específica.

Se deduce de las cifras consideradas que el médico general tiene "olfato" para remitir a urgencias los enfermos con apendicitis, bien con el diagnóstico probable, bien con otro diagnóstico, o sin diagnóstico. Efectivamente, el médico general “sabe” mucho más de lo que le parece, como sucede con todos los médicos ${ }^{17}$. Comete errores entre los que sospecha que tienen apendicitis (falsos positivos) y entre los que deriva por otros diagnósticos (falsos negativos), pero al final remite a todos los que tienen apendicitis, con una u otra etiqueta. Y, sobre todo, mantiene a la amplia mayoría de sanos (verdaderos negativos) lejos de los especialistas. Por ello es clave la función de filtro del médico general.

\section{El valor predictivo de las pruebas diagnósticas}

Es prueba diagnóstica la intervención que introduce un cambio en nuestro conocimiento del problema del paciente y con ello reduce la incertidumbre en la asignación de un diagnóstico, con todo lo que ello significa respecto a pronóstico y tratamiento (a la toma de decisiones). Desde este punto de vista, son pruebas diagnósticas no sólo las 
pruebas analíticas, de imagen o instrumentales en general, sino también los datos de la historia clínica, y de la exploración física. Por ejemplo, la expresión del paciente acerca de la presencia e intensidad del do$\operatorname{lor}^{18}$. Incluso es prueba diagnóstica, si se entiende en su amplio sentido, el conocimiento epidemiológico acerca de la incidencia y prevalencia de la enfermedad en el ámbito en que se atiende al paciente.

Las pruebas diagnósticas permiten disminuir la incertidumbre respecto al diagnóstico, si se aplican juiciosamente. El médico general acumula información de sus pacientes, lo que le permite revisar las sospechas diagnósticas iniciales y la probabilidad que asigna a las mismas ${ }^{2}$. La nueva información diagnóstica sirve para revisar probabilidades hasta que sea más ventajoso parar el proceso y pasar a la acción (tratar, o esperar en una "espera expectante"), cuando se ha llegado al "umbral terapéutico" 19 . La información que da la prueba, su beneficio diagnóstico, depende de dos características: (1) de la bondad de la prueba (la sensibilidad y la especificidad de la prueba diagnóstica), y (2) de la probabilidad que tiene el paciente de presentar el diagnóstico que se sospecha (la prevalencia de la enfermedad en el ámbito en que se atiende al paciente, si no hay mejor información). Respecto a la segunda característica, en cada nuevo problema de salud el médico general puede valorar a muy bajo costo esta probabilidad pre-prueba por el conocimiento acumulado acerca del paciente, su familia y su comunidad, por ofrecer longitudinalidad ${ }^{2}$. Este acúmulo de información representa el resultado de muy sutiles "pruebas diagnósticas" no instrumentales, que permiten disminuir la incertidumbre diagnóstica antes de solicitar pruebas diagnósticas instrumentales.

La probabilidad pre-prueba de una enfermedad es equivalente a la prevalencia de la misma en la población de que procede el paciente, cuando no se sabe nada más. Naturalmente, la probabilidad pre-prueba se refina con el conocimiento del paciente, bien acumulado por la longitudinalidad, bien por el proceso de valoración, a través de la entrevista clínica y de la exploración física.

No hay pruebas diagnósticas perfectas, $y$ el resultado de cada una añade información y contribuye a disminuir la incertidumbre, pero no la elimina radicalmente. Un resultado positivo no asegura que el paciente tenga la enfermedad, ni un resultado negativo la excluye. La información que da la prueba es una pieza más, pero raramente es definitiva. La probabilidad de enfermedad después de la prueba se valora con los valores predictivos, negativo y positivo ${ }^{\mathrm{e}}$. Un resultado positivo aumenta la probabilidad de que el paciente tenga la enfermedad, pero no excluye por completo que esté sano. Un resultado negativo aumenta la probabilidad de que el paciente no tenga la enfermedad, pero no excluye completamente que la tenga.

Los valores predictivos dependen de (1) la sensibilidad y especificidad de la prueba, $\mathrm{y}$ (2) de la probabilidad pre-prueba de enfermedad [de la prevalencia de la enfermedad entre los pacientes de ese ámbito sanitario, si no hay más información]. La prevalencia de la enfermedad modifica grandemente el valor predictivo de los resultados de una prueba.

En teoría, con una prueba diagnóstica dada (de sensibilidad 95\% y especificidad $90 \%$, por ejemplo) el valor predictivo positivo sube del $9 \%$ hasta el $51 \%$ según se aplique a pacientes de un ámbito en el que la prevalencia de enfermedad sea de $1 \%$ o del $10 \%$.

En un caso práctico, del diagnóstico de fiebre mediante el tacto del médico, el valor predictivo positivo subió del $8 \%$ al $69 \%$ según se aplicase a una población de pacientes con prevalencia de fiebre del $1 \%$ y del $20 \%$, respectivamente. Se encuentran las mismas cifras para el diagnóstico de la anemia por la

eEn su juicio diagnóstico los médicos generales, por el ámbito en el que trabajan, tienen gran poder predictivo negativo, y si deciden que un paciente está sano lo más probable es que el paciente esté sano. Por el contrario, los especialistas tienen gran poder predictivo positivo, por el ámbito en el que trabajan, y si deciden que un paciente está enfermo lo más probable es que el paciente esté enfermo. 
valoración de la palidez conjuntival. Respecto al valor predictivo negativo, se mantuvo alto con dichas pruebas diagnósticas en ambas poblaciones, en torno y más del $90 \%^{20}$.

El trabajo concatenado del médico general y del especialista facilita que tome contacto con el segundo nivel un grupo seleccionado de pacientes cuya prevalencia de enfermedad es mucho mayor que en el grupo sin seleccionar que toma contacto con el médico general. Con ello se potencia el alto valor predictivo positivo que tiene el trabajo de los especialistas en su ámbito. Por ejemplo, respecto al dolor abdominal, hay datos empíricos que demuestran que la prevalencia de "necesidad de cuidados especializados" pasa del $7 \%$ en la consulta del médico general, al $59 \%$ en los pacientes derivados a atención especializada ${ }^{13}$. En el mismo sentido, la prevalencia de apendicitis aguda pasa del $1 \%$ entre los pacientes que consultan al médico general por dolor abdominal, al 20\% entre los que se derivan a urgencias hospitalarias $^{13,15}$. En lo que se refiere al dolor precordial, la prevalencia de enfermedad coronaria pasa del $3 \%$ en la consulta del médico general de una iguala, al $72 \%$ en los pacientes derivados para angiografía ${ }^{21}$.

El médico general parte de una cesta de manzanas con muchas sanas, y selecciona muy adecuadamente a las verdaderamente sanas (verdaderos negativos), dado el ámbito en el que trabaja, por su alto cociente de verosimilitud negativo, y su alto valor predictivo negativo [no se puede excluir, claro, que se quede con alguna manzana podrida, falso negativo]. En el ámbito de la atención primaria, la prevalencia de enfermedad es baja, y el médico general asigna bien la probabilidad pre-prueba, por su conocimiento acumulado a través de la longitudinalidad (con ello palia, en parte, lo poco que significa ser un caso clínico “típico" en primaria, si se emplean criterios de especialistas).

El médico general deriva a los especialistas cajas de manzanas acordes a las habilidades y conocimientos de cada tipo de especialista, con alguna manzana sana (falso positivo), y con muchas podridas (verdaderos positivos). Es decir, el médico general selecciona los pacientes a derivar, y la especialidad a la que derivar a cada paciente. $Y$ puede cometer errores tanto respecto a la selección de los pacientes, como a la elección de la especialidad.

El especialista, con el grupo seleccionado que deriva el generalista, tiene un alto cociente de verosimilitud positivo y un alto valor predictivo positivo, por lo que determina con precisión las manzanas que están enfermas ly a veces considera como enferma alguna que en realidad está sana, lo que le expone a técnicas diagnósticas innecesarias hasta que se aclara su estado de buena salud]. En el ámbito de la atención especializada, la prevalencia de la enfermedad es alta, y los casos clínicos "típicos" lo son en todo su sentido, puesto que se definen en los libros al uso, y en la enseñanza pre y postgraduada de acuerdo a la experiencia de los pacientes en el ámbito hospitalario. Por todo ello, si el especialista recibe un grupo altamente seleccionado y con enfermedades de su especialidad, se facilita el rendimiento y la adecuación de su estrategia diagnóstica.

Es crucial, pues, que el médico general tenga la función de filtrar los pacientes que llegan a la atención especializada, con lo que aumenta extraordinariamente el valor predictivo positivo de las pruebas diagnósticas que aplica el especialista. No es sólo filtrar para aumentar la prevalencia en los pacientes derivados, sino seleccionar adecuadamente a los pacientes para que le sean beneficiosos los conocimientos y habilidades del tipo de especialidad seleccionada. Es, pues, desembarcar a los pacientes desde el Mar de la Incertidumbre (1) cuando lo requieren y (2) el en Puerto oportuno de la Tierra de los Síntomas ${ }^{9}$.

\section{Algunos ejemplos prácticos del filtro personal y familiar y del médico general}

Hay varios ejemplos en los que se puede analizar la conducta de consulta de la población y la conducta de derivación de los médicos generales. Así, respecto a la rectorragia, la sangre en heces, tiene en la población una 
incidencia anual del $20 \%$. Sólo consulta con el médico general el $4 \%$ de estos pacientes. El médico general suele resolver la mayor parte de las rectorragias, tranquilizando al paciente, con la exploración, las pruebas y recomendaciones y tratamientos pertinentes, y deriva al especialista, anualmente, aproximadamente el $14 \%$ de los pacientes que le consultan con rectorragia ${ }^{3,4}$.

Es decir, en un año, de 400 personas con un episodio de rectorragia (en una población de 2.000 personas en total), consultan 16 con el médico general, y van al especialista 2. Así se "concentra" la prevalencia del cáncer de recto y del sigma. Dicha prevalencia es del $0,1 \%$ en la población general con rectorragia, del $2 \%$ entre los que consultan con el médico general, y del $36 \%$ entre los que se derivan ${ }^{3,4}$.

Es decir, 1/ el filtro familiar y personal multiplica por 20 la prevalencia del cáncer de recto y de sigma y 2 / el filtro del médico general multiplica por 18 dicha prevalencia. Como resultado, el especialista recibe un grupo de pacientes con 360 veces la prevalencia de cáncer de recto y de sigma en la población general con rectorragia. Así se mejoran los resultados y se justifican las técnicas diagnósticas de los especialistas.

Esta conducta que lleva al aumento de la prevalencia de enfermedad en los distintos grupos de pacientes no está bien estudiada. Ni sabemos qué provoca la consulta con el médico general de un paciente con rectorragia, ni cómo elige el médico general a los pacientes para derivar ${ }^{3,4}$. Parece que la mayor edad y la sangre mezclada con heces son dos características básicas en este proceso.

En cualquier caso, es evidente la eficacia del filtro, que también se demuestra respecto a otros casos ya considerados, como la apendicitis en el dolor abdominal agudo, que pasa de una frecuencia en la consulta del médico general del $1 \%$ a una prevalencia del $20 \%$ en urgencias [curiosamente, sólo la mitad de los pacientes llegan adecuadamente etiquetados como "sospecha de apendicitis"] ${ }^{13,15}$. O el dolor torácico de causa coronaria, que pasa del $3 \%$ en la consulta de primaria al $72 \%$ antes de la angiografía ${ }^{21}$.
El filtro es, además, muy importante en ocasiones especiales, como cuando existe una alarma sanitaria. Sirve de ejemplo la epidemia del SARS y su efecto sobre los servicios sanitarios en Toronto (Canadá) ${ }^{22}$. Por cada caso confirmado hubo 10 casos potenciales, 100 contactos y 1.400 sanos preocupados que llegaron a consultar. Sin un filtro adecuado, la demanda sería incontrolable.

Sería incontrolable la demanda con problemas muy frecuentes, como el dolor lumbar, que tiene una prevalencia anual en la población del $40 \%$. Sólo consulta con el médico general el $12 \%$ de estos pacientes. El médico general suele resolver la mayor parte de los dolores lumbares, con la exploración, recomendaciones y tratamientos adecuados, y deriva al especialista anualmente el $6 \%$ de estos pacientes. Aproximadamente se solicita radiología al $12 \%$ [un exceso, que medicaliza el problema ${ }^{23,24}$. Es decir, en un año, de 800 personas con un episodio de dolor lumbar (en una población de 2.000), consultan 90 con el médico general, y van al especialista 5 .

¿Cómo afecta la gravedad a la derivación?. Los hechos demuestran, como es lógico, que la tasa de derivación aumenta con la gravedad y el deterioro de la calidad de vida, y ello afecta a los dos filtros, el personal y familiar y el del médico general. Por ejemplo, en el caso de la incontinencia urinaria, encontramos una prevalencia del $30 \%$ en la población femenina de más de 20 años pero sólo consulta con el médico general el $5 \%$ de estas pacientes. El filtro según gravedad se establece fundamentalmente a nivel personal y familiar, pues si se da valor de 1 a la frecuencia de toma de contacto con el médico general por las mujeres con incontinencia leve, dicha frecuencia se multiplica por 5,7 en casos moderados y 14 en casos graves. La probabilidad de derivación por el médico general al especialista fue de 1,1 y 1,2 respectivamente para casos moderados y graves en comparación con los leves, con un peso importante en la decisión de derivar de la edad de la mujer (se derivó más a las mujeres más jóvenes). Así, la frecuencia relativa de llegar al especialista desde la comunidad 
según gravedad, es de 5,9 y 16,4 veces para las mujeres con incontinencia moderada y grave por comparación con las leves ${ }^{25}$. El médico general, pues, se fía del criterio de gravedad propio de la población femenina con incontinencia urinaria y, una vez que la paciente le consulta, su criterio de derivación "coincide" con el previo y lo matiza sobre todo por la edad de la paciente. Quizá ello signifique tanto el acierto de la población femenina como que el médico general no domina las técnicas diagnósticas ni terapéuticas al respecto, pese a que son simples, sencillas, eficaces y de fácil aplicación en atención primaria.

\section{Cuestiones críticas en torno al papel de filtro del médico general}

El caso de la incontinencia urinaria en la mujer sirve para plantear el problema general de los conocimientos, actitudes y habilidades que precisa el médico general para filtrar adecuadamente la demanda. Sabemos muy poco sobre los criterios con los que selecciona el médico a determinados pacientes para derivarlos a determinados especialistas, aunque está demostrada la utilidad del filtro, al menos en casos concretos como en la rectorragia ${ }^{3}$. También sabemos que el médico general deriva más a los pacientes con enfermedades infrecuentes, o a los pacientes con enfermedades frecuentes con comorbilidad, más complejos ${ }^{26}$. Pero nuestro conocimiento es escaso acerca:

- de la evolución natural de las enfermedades en general,

- del largo camino que transcurre entre el inicio de los síntomas y la decisión de consultar al médico general,

- del proceso diagnóstico que lleva al médico general a derivar al paciente, en su caso (con errores y aciertos), y

- de la resolución del problema con la ayuda del especialista (con errores y aciertos) $)^{5,10,13,14,25-29}$.

Lo fundamental es que el médico general derive juiciosamente, ni en exceso (se formarían colas con pacientes esperando para recibir cuidados innecesarios de los espe- cialistas, y se disminuiría la prevalencia de enfermedades entre los pacientes que pasan al nivel secundario con lo que no se justificarían su técnicas diagnósticas), ni en defecto (habría retraso o ausencia de cuidados necesarios, con repercusión en la salud y el coste sanitario), pero no están claros ni las fronteras ni los roles de los especialistas ${ }^{28,29}$.

Respecto al filtro del médico general en el sistema sanitario, hay dos cuestiones críticas que se asumen sin discutir:

- se precisan niveles en la organización de los sistemas sanitarios. Es decir, $i$ son necesarios los niveles en que trabajan médicos generales y especialistas?; pudiera ser que no fueran necesarios.

- el acceso directo de los pacientes a los especialistas conlleva el uso adecuado de los recursos especializados. Tener certeza en esta cuestión exige responder a: ¿puede el filtro personal y familiar de pacientes bien informados tener un rendimiento comparable al del médico general que trabaja adecuadamente? ${ }^{9}$.

Se suele aceptar que el médico general elige especialista mejor que el paciente bien informado. Ello se ha demostrado respecto al mejor uso de las urgencias en Europa, también en España. En EE.UU., varios estudios sobre el efecto del filtro (allí con un uso puramente económico) han demostrado su eficacia en el control del gasto, pero con resultados contradictorios acerca de su impacto sobre la adecuación del uso de urgencias ${ }^{30}$. En Europa, al menos, los médicos generales españoles seleccionan mejor que los propios pacientes quién deber ir a urgencias, y así hay más consultas de urgencia adecuada entre los derivados por el médico general que entre los que acuden espontáneamen$\mathrm{te}^{31-33}$. En otro ejemplo, en la consulta por dolor torácico en urgencias en un estudio belga, la proporción de angina inestable fue del $9 \%$ entre los pacientes que acudieron espontáneamente y del $15 \%$ en los derivados por su médico general; la proporción de pacientes con causa locomotora de su dolor fue de 8 y $6 \%$ respectivamente ${ }^{34}$.

Es importante hacer notar que los médicos generales pueden mejorar la utilización 
de las urgencias no sólo a través de su mejor criterio al derivar, sino también a través de un mejor control de los pacientes "grandes utilizadores". Este grupo de pacientes concentra el uso de los servicios sanitarios, y se ha demostrado su menor uso de urgencias (y la disminución de los ingresos consiguientes) con el simple envío al médico general de un listado trimestral con los nombres de estos pacientes ${ }^{35}$. Así pues, lo importante no es sólo el papel de filtro, sino el buen trabajo global.

La adecuada función de filtro tiene fundamento científico teórico y práctico, pero exige de los médicos generales una gran ca- pacitación y actualización de sus conocimien-

tos y una actitud de prestación de servicios

que asegure la eficiencia de los cuidados a través de una alta capacidad resolutiva.

Cuanto mejor sea el servicio global que ofrezca el médico general más se justificará su papel de filtro ${ }^{2,27}$.

\section{Nota}

Este texto se basa en un capítulo del libro que están escribiendo los autores sobre "Reivindicación de una medicina clínica cercana, científica y humana".

\section{Bibliografía}

1. Gérvas J, Ortún V. Caracterización del trabajo asistencial del médico general/ de familia. Aten Primaria 1995; 16: 501-6.

2. Ortún V, Gérvas J. Fundamentos y eficiencia de la atención médica primaria. Med Clín (Barc) 1996; 106: 97-102.

3. Fijten GH, Blijham GH, Knottnerus JA. Ocurrence and clinical significance of overt blood loss per rectum in the general population and medical practice. Br J Gen Pract 1994; 44: 320-5.

4. Crosland A, Jones R. Rectal bleeding: prevalence and consultation behaviour. BMJ 1995; 311: 486-8.

5. Pérez Fernández M, Gérvas J. El efecto cascada: implicaciones clínicas, epidemiológicas y éticas. Med Clín (Barc) 2002; 118: 65-7.

6. Horder J, Horder E. Illness in general practice. Practitioner 1954; 173: 177-87.

7. White KL, Williams FT, Greenberg BG. The ecology of medical care. N Engl J Med 1961; 265: 885-92.

8. Knottnerus JA. Medical decision making by general practitioners and specialists. Fam Pract 1991; 8: 305-7.

9. Gérvas J, Pérez Fernández M. Aventuras y desventuras de los navegantes solitarios en el Mar de la Incertidumbre. Aten Primaria 2005; 35: 95-8.

10. Flórez M, Valverde MD, García F. Historia natural de la hernia de disco lumbar. Rehabilitación 1993; 27: 35764.

11. Forés M, Gérvas J, Bonet M, Marcos L, Tomás P, Sagués A et al. ICPC in Spain. Epidemiological aspects of patient data in Spanish general practice. En: The International Classification of Primary Care in The European Community, with a multi-language layer. Lamberts H, Wood M, Hofman I (eds). Oxford: OUP; 1993, págs. 119-24.
12. Muris JWM, Starmans R, Fijten G, Knottnerus JA. Oneyear prognosis of abdominal complaints in general practice: a prospective study of patients in whom non organizc cause is found. Br J Gen Pract 1996; 46: 715-9.

13. Edwards MW, Forman WM, Walton J. Audit of abdominal pain in general practice. J R Coll Gen Pract 1985; 35: 235-8.

14. Muris JWM, Starmans R, Fijten GH, Crebolder HFJM, Krebber TPWA, Knottnerus JA. Abdominal pain in general practice. Fam Pract 1993; 10: 387-400.

15. Simmen HP, Decurtins M, Rotzer A, Duff C, Brütsch HP, Largiadèr F. Emergency room patients with abdominal pain unrelated to trauma: prospective analysis in a surgical university hospital. HepatoGastroenterol 1991; 38: 279-82.

16. Beasley SW. Can we improve diagnosis of acute appendicitis?. BMJ 2000; 321; 907-8.

17. Tanenbaum S. What physicians know. $N$ Engl J Med 1993; 329: 1268-71.

18. Knottnerus JA, Weel CW, Muris WM. Evaluation of diagnostic procedures. BMJ 2002; 324: 477-80.

19. Pauker SG, Kassirer JP. The threshold approach to clinical decision making. $N$ Engl J Med 1980; 302: 110917.

20. Valeiras C, Calvo A, Roca A, Gude F, González A. Valor del tacto en el diagnóstico de fiebre, y de la palidez en el diagnóstico de anemia. Estudio observacional en adultos. An Med Interna 2002; 19: 120-5.

21. Sox HC, Hickam DH, Marton KI, Moses L, Skeff KM et al. Using the patient's history to estimate the probability of coronary artery disease: a comparison of primary care and referral practices. Am J Med 1990; 89: 7-14. 
22. Weinstein RA. Planning for epidemics. The lessons of SARS. $N$ Engl J Med 2004; 350: 232-3.

23. Schers H, Bradspenning J, Drijver R, Wensing M, Grol R. Low back pain in general practice: reported management and reasons for not adhering to the guidelines in the Netherlands. Br J Gen Pract 2000; 50: 640-4.

24. Dey P, Simpson CWR, Collins SI, Hodgson G, Dowrick $\mathrm{CF}$, Simison AJM et al. Implementation of RCGP guidelines for acute low back pain: a cluster randomised controlled trial. Br J Gen Pract 2004; 54: 337.

25. Hunskaar S, Seim A, Freeman T. The journey of incontinent women from community to university clinic; implications for selection bias, gatekeeper function and primary care. Fam Pract 1996; 13: 363-8.

26. Forrest C, Reid RJ. Prevalence of health problems and primary care physicians' speciality referral decisions. $J$ Fam Pract 2001; 50: 427-32.

27. Green C, Holden J. Diagnostic uncertainty in general practice. A unique opportunity for research?. Eur J Gen Pract 2003; 9: 13-5.

28. García Mariñoso B, Jelovak J. GP’ payment contracts and their referral practice. J Health Economics 2003; 22: 617-35.
29. Starfield B. Research in general practice: co-morbidity, referrals and the role of general practitioners and specialists. SEMERGEN 2003; 23 (supl 1): 7-13.

30. Peiró S, Sempere T, Oterino D. Efectividad de las intervenciones para reducir la utilización inapropiada de los servicios hospitalarios de urgencias. Revisando la literatura 10 años después del Informe del Defensor del Pueblo. Bol Economía Salud 1999; 33: informe técnico no 2 .

31. Sánchez M, Molina JC, Torre M, Muñoz F, Rodríguez J, González A, Cánovas J. La asistencia pediátrica vista a través de un servicio de urgencias pediátricas. Rev San Hig Pub 1990; 64: 283-301.

32. Antón MD, Peña JC, Santos R, Sempere E, Martínez J, Perula LA. Demanda inadecuada a un servicio de urgencias pediátrico hospitalario: factores implicados. Med Clín (Barc) 1992; 99: 743-6.

33. Vilardell L. ¿Por qué ingresan los pacientes de forma urgente en un hospital?. Med Clín (Barc) 1994; 103: 543-53.

34. Buntinx F, Knockaert D, Bruyninckx R, Blaey N, Aerts $\mathrm{M}$, Knottnerus JA et al. Chest pain in general practice or in the hospital emergency department: is it the same? Fam Pract 2001; 18: 586-9.

35. Brandon WR, Chambers R. Reducing emergency department visits among high-using patients. J Fam Pract 2003; 52: 637-40. 